\title{
A missed ureteral injury secondary to aortic aneurysm surgery: could a correct diagnostic approach avoid serious complications?
}

\begin{abstract}
Ureteral injuries represent a complication during retroperitoneal and pelvic surgery; in particular, gynaecologic surgery is at the first place among causes of ureteral lesions. However, another cause of ureteral injuries could be associated with vascular surgery. We describe a case of a missed ureteral injury after an elective operation of aortic aneurysm repair with caused very serious complications in a patient in good health status. A correct diagnostic approach could avoid serious complications helping to reduce hospitalization time.
\end{abstract}

Volume 4 Issue $3-2017$

\author{
Giovanni Luca Giliberto, Carmelo Agostino \\ Di Franco, Silvia La Vecchia, Bruno Rovereto \\ Department of Urology, University Hospital IRCCS Policlinico S \\ Matteo-Pavia, Italy
}

Correspondence: Giovanni Luca Giliberto, Department of Urology, University Hospital IRCCS Policlinico S Matteo-Pavia, Italy, Email gl.giliberto@gmail.com

Received: January 13, 2017 | Published: March 31, 2017

\section{Introduction}

Overall, the incidence of iatrogenic ureteral injuries is reported $0,05-30 \%$, in relation to surgeons experience. ${ }^{1,2}$ Ureteral injury is one of the most serious complications during retroperitoneal and pelvic surgery. In particular, gynecologic procedures are often associated with ureteral injuries and this, if not recognized, can be associated with significant morbidity, such as ureterovaginal fistulas or the potential loss of renal function, especially in late diagnosis. In literature, ureteral iatrogenic damages are reported in association with abdominal hysterectomy in particular ligation of uterine and ovarian vessels, radical hysterectomy, and abdominal perineal resection. . $^{3,4}$ Ureter is injured in $0.5-2 \%$ of all abdominal hysterectomies and routine gynaecological pelvic operations whereas in 5-30\% of radical hysterectomies. ${ }^{5}$ Ureteral injuries are less common during vascular surgery, approximately $5-15 \%$ of cases. ${ }^{2}$ Diagnosis is fundamental to prevent serious complications. We present a case of ureteral damage diagnosed three weeks after planned repair of abdominal aortic aneurysm. Delayed diagnosis caused serious complications avoidable with an early correct diagnosis.

\section{Case report}

A 54-years old Caucasian man affected by sub renal aortic aneurysm, undertake vascular surgery of aortotomy and bilateral aortoiliac Dacron prosthesis implantation. Patient had a good health status; he had hypertension (with good control of blood pressure), diabetes mellitus type 2 . He had no operations. In the past. In 5 post-operative days, due to post-operative anaemia, fever and slight increased creatinine (around $1.3 \mathrm{mg} / \mathrm{dl}$ ), the patient received an ultrasound scan that showed intra-retroperitoneal fluid without any sign of hydronephrosis. In the suspect of haemorrhage, vascular surgeons performed an abdomen CT-scan with contrast that reported organized hematoma along the right iliac branch associated to intraperitoneal serous-hematic fluid (Figure 1). CT-scan did not have delayed phases. In XII post-operative day, patient was transferred to intensive care ward because of fever until $39^{\circ} \mathrm{C}$, dynamic ileus and uremic status. He received an abdomen contrast CT-scan that showed increasing of intra-retroperitoneal fluid with small bowel and right colon compression and increase of right iliac region serous-hematic fluid (Figure 2).

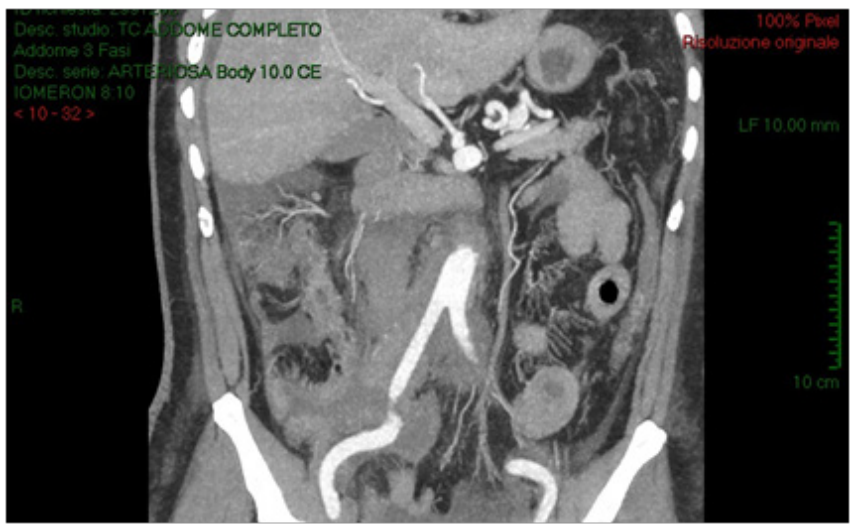

Figure I Abdomen CT-scan with contrast that reported organized hematoma along the right iliac branch associated to intraperitoneal serous-hematic fluid. 


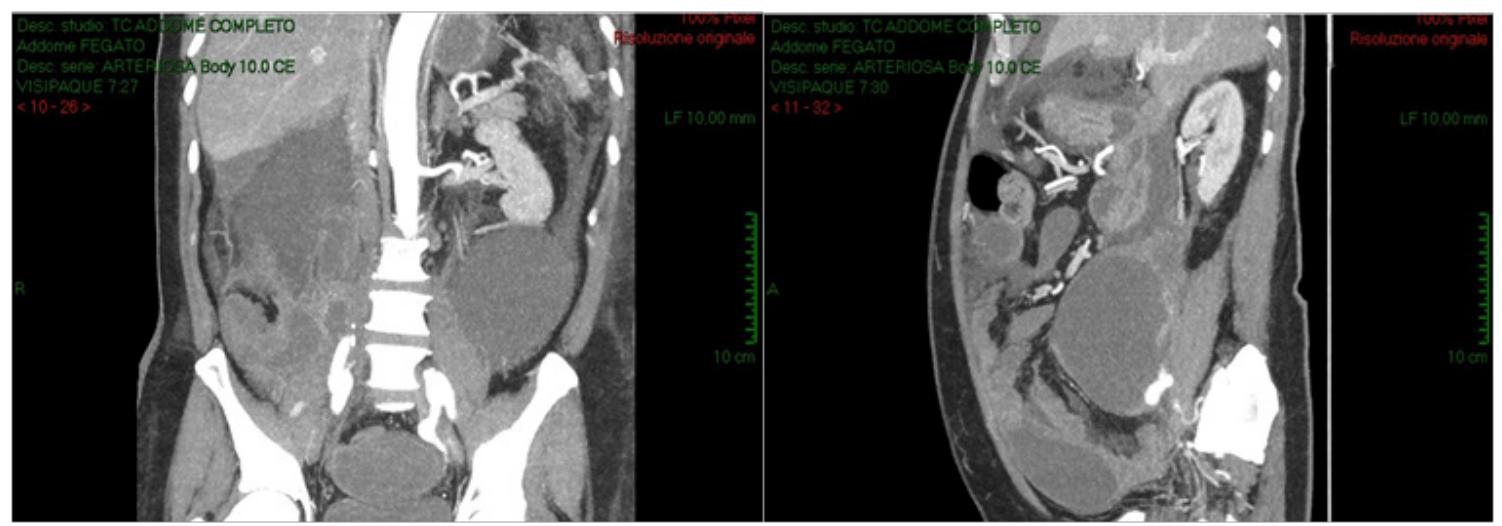

Figure 2 CT-scan shows increasing of intra-retroperitoneal fluid with small bowel and right colon compression and increase of right iliac region serous-hematic fluid.

The CT-scan, targeted to a suspect of hemorrhage, did not have delayed phases. Patient received paracentesis with around $3200 \mathrm{cc}$ fluid drained that was sent to microbiological examination. Result of bacteriological exam revealed infection by Proteus Mirabilis and he received target antibiotic therapy with resolution of septic status. In XXII post-operative day, for a progressive increase of serum creatinine we decided to perform an Uro-CT scan. The exam showed reduction of intraperitoneal fluid, right iliac side fluid collection with contrast enhancement during delayed phases; right ureter was not visible under fluid collection (Figure 3). We attempted to insert a ureteral stent in retrograde way but ureter resulted totally interrupted inside the fluid collection so the patient was undergone to explorative laparotomy and toilette of fluid collections. We found ureteral loss of substance of around 3 centimeter localized in anterior ureteral wall. We manufactured end-to-end ureteral anastomosis after positioning of ureteral stent seven French. We removed ureteral stent after 3 months and currently the patient is in follow-up with a good renal function as shown at last CT-scan after 10 months (Figure 4).

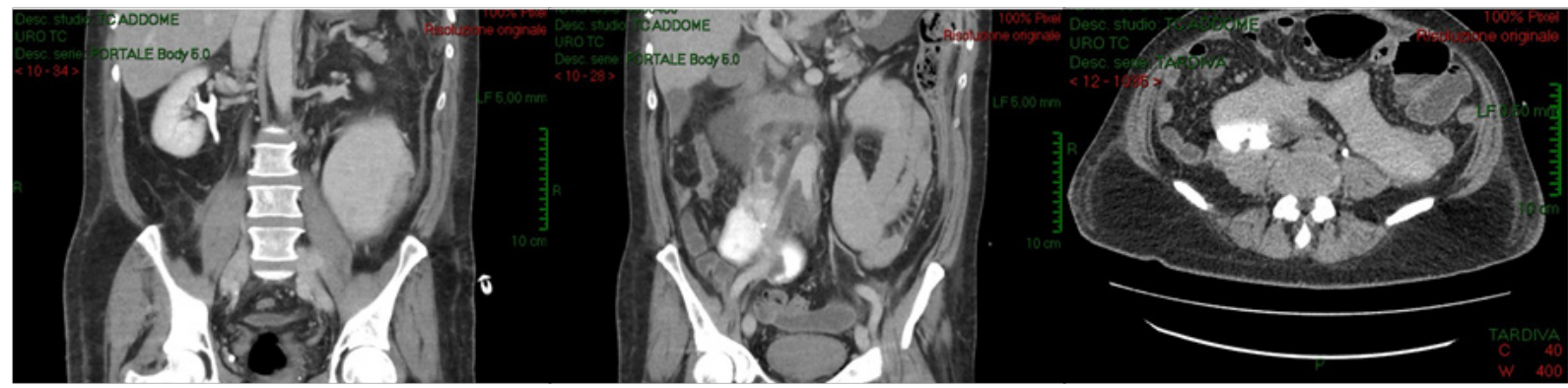

Figure 3 Post paracentesis Uro-CT scan showing reduction of intraperitoneal fluid, right iliac side fluid collection with contrast enhancement during delayed phases; right ureter was not visible under fluid collection.
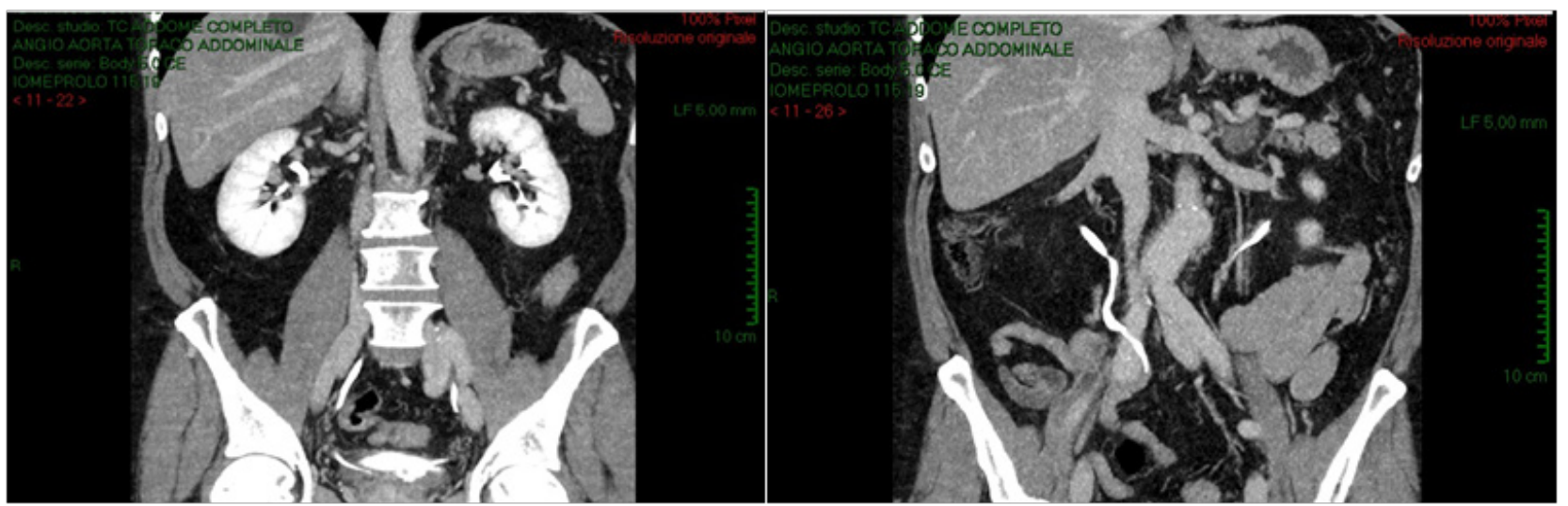

Figure 4 After ureteral stent removal, right ureter appears normal ducted with a good kidney function. 


\section{Discussion}

Ureteral damages during open surgery can be caused by ligation or laceration; moreover stripping of ureteral vessels may result in ischemia and subsequent fistula or stricture evolution. ${ }^{4}$ During vascular surgery, ureteral injury is a complication to fear and if it occurs, it is usually associated to periaortic inflammatory fibrosis or retroperitoneal haemorrhage. ${ }^{6}$ In fact, it is important to suspect a ureteral injury when a patient, who underwent surgery potentially "hazardous" to the ureter, has an anomalous post-operative course. Fever, infectious signs, dynamic ileus, renal failure due to re-absorption of urine and metabolites may characterize clinical presentation of ureteral leaks. Hematuria is not always present; in fact, it occurs in up to $30 \%$ of cases. ${ }^{7}$ If ureteral lesion is not identified and treated, it may evolve in serious complication that endanger the patient's life, as we describe in this case, in which patient needed intensive care support. Imaging is very important in the diagnosis of ureteral damages, in particular CT-scan is the most appropriate exam. However, CT-scan must be performed with contrast, to exclude possible causes of bleeding or hematoma, but CT-scan delayed phases must be performed. Delayed CT-scan images may demonstrate contrast extravasation in ureteral leaks or absence of contrast excretion in stenosis or extrinsic compression for example by hematoma or abscess. When diagnosis is done, treatment consists, if possible, in placement of ureteral double J stent. Proximal and mid-ureteral leakages may be treated with endto-end ureteral anastomosis, while when loss of substance is localized in distal part, can be required ureteroneocystostomy with psoas hitch. ${ }^{8}$ In some cases, when ureteral lesion is limited, percutaneous nephrostomy may allow spontaneous resolution of leakage. ${ }^{9}$

\section{Conclusion}

Missed ureteral injuries during or after abdominal surgery can be associated with serious complications. A correct diagnosis is fundamental and in these cases a CT-scan with delayed post-contrast sequences can help in differential diagnosis to early identify damages to urinary tract.

\section{References}

1. Brandes S, Coburn M, Armenakas N, et al. Diagnosis and management of ureteric injury: an evidence-based analysis. BJU Int. 2004;94(3):277-289.

2. Selzman AA, Spirnak JP. Iatrogenic ureteral injuries: a 20-year experience in treating 165 injuries. J Urol. 1996;155(3):878-881.

3. Aslan P, Brooks A, Drummond M, et al. Incidence and Management of Gynaecological-Related Ureteric Injuries. Aust $N$ Z J Obstet Gynaecol. 1999;39(2):178-181.

4. Dunnick, Reed, Carl Sandler, Jeffrey Newhouse. Textbook of uroradiology. Lippincott Williams \& Wilkins, USA. 2012.

5. Daly JW, Higgins KA. Injury to the ureter during gynecologic surgical procedures. Surg Gynecol Obstet. 1988;167(1):19-22.

6. Adams JR, Mata JA, Culkin DJ, et al. Ureteral injury in abdominal vascular reconstructive surgery. Urology. 1992;39(1):77-81.

7. Campbell EW, Filderman PS, Jacobs SC. Ureteral injury due to blunt and penetrating trauma. Urology. 1992;40(3):216-220.

8. Fry DE, Milholen L, Harbrecht PJ. Iatrogenic ureteral injury: options in management. Arch Surg. 1983;118(4):454-457.

9. Lask D, Abarbanel J, Luttwak Z, et al. Changing trends in the management of iatrogenic ureteral injuries. J Urol. 1995;154(5):1693-1695. 